Int. J. Dev. Biol. 55: 495-503

doi: $10.1387 / \mathrm{ijdb} .103227 \mathrm{sn}$

\title{
The interplay between macrophages and angiogenesis in development, tissue injury and regeneration
}

\author{
SILVIA NUCERA ${ }^{1,2,3}$, DANIELA BIZIATO ${ }^{1,2,3}$ and MICHELE DE PALMA*,1,2 \\ ${ }^{1}$ Angiogenesis and Tumor Targeting Research Unit, ${ }^{2}$ San Raffaele-Telethon Institute for Gene Therapy (HSR-TIGET), \\ Division of Regenerative Medicine, Stem Cells and Gene Therapy, San Raffaele Scientific Institute, Milan, Italy and \\ ${ }^{3}$ Vita-Salute San Raffaele University Medical School, Milan, Italy
}

\begin{abstract}
During organ development and remodeling, macrophages support angiogenesis, not only by secreting proangiogenic growth factors and matrix-remodeling proteases, but also by physically interacting with the sprouting vasculature to assist the formation of complex vascular networks. Recent data further indicate that embryonic and tumor-associated macrophages express similar genetic programs, possibly suggesting convergent functions in organogenesis and tumorigenesis. In this article, we review the role of macrophages in development, tissue injury and regeneration, by focusing on the mechanisms used by subsets of these cells, such as the TIE2-expressing macrophages, to regulate angiogenesis and lymphangiogenesis in both fetal and post-natal life.
\end{abstract}

KEY WORDS: monocyte, lymphangiogenesis, wound healing, tumor, tumor-associated macrophage

\section{The monocyte/macrophage system}

In adulthood, monocytes are short-lived cells that derive from bone marrow (BM) hematopoietic stem cells (HSCs) and circulate in the peripheral blood. In humans, monocytes represent $5-8 \%$ of all circulating leukocytes. Under inflammatory conditions, monocytes extravasate and migrate into tissues, where they differentiate into macrophages and dendritic cells (DCs). It is now clear that circulating monocytes are a heterogeneous cell population, which may comprise distinct subsets with distinguishing phenotypic and functional features (Geissmann et al., 2010). Circulating monocytes can be conveniently identified by their physical properties (light scattering) and expression of colony-stimulating factor-1 receptor (CSF-1R, or CD115). Based on their phenotype (surface marker profile and gene expression) and functions, both mouse and human monocytes can be divided into at least two main subsets, which have been termed "inflammatory" or "classical" monocytes $\left(\mathrm{Gr} 1^{+}\right.$/ Ly- $6 C^{\text {high }}$ in the mouse; CD14 ${ }^{+}$CD 16 - in the human), and "resident", "patrolling" or "non-classical" monocytes (Gr1-/Ly6Clow/- in the mouse; CD14 ${ }^{\text {low/-CD16+ }}{ }^{+}$in the human), respectively (Geissmann et al., 2010; Ziegler-Heitbrock et al., 2010). A third subset has been described that displays intermediate features between the two main subsets (Geissmann et al., 2010; Ziegler-Heitbrock et al., 2010). Whereas "inflammatory" monocytes participate in inflammatory responses induced by pathogens or tissue damage, and can rapidly differentiate into inflammatory macrophages or
DCs upon their extravasation, the role of "resident" monocytes is still poorly understood. Recent studies have suggested that "resident" monocytes patrol blood vessels (hence "patrolling" monocytes) and extravasate in response to vascular damage to facilitate tissue healing and revascularization (Auffray et al., 2007; Nahrendorf et al., 2007).

Both in development and adulthood, tissue macrophages phagocytose pathogens, dead cells, cellular debris, and various components of the extra-cellular matrix (ECM). Ilya llyich Mechnikov was the first to describe the process of phagocytosis in 1882, and macrophages were named after this feature as "big eaters" (from ancient Greek, makros "large" + phagein "eat"). However, it is now clear that macrophages are not only big eaters of pathogens and dead cells, but also important components of the stromal architecture of several tissues and organs, where they regulate organ homeostasis and remodeling (Pollard, 2009). For instance, Kupffer cells are specialized macrophages that line hepatic capillaries (termed sinusoids) in the liver, where they scavenge senescent erythrocytes, a process referred to as hemocatheresis. During development and tissue healing or regeneration, macrophages

Abbreviations used in this paper: BM, bone marrow; CSF, colony-stimulating factor; DC, dendritic cell; EC, endothelial cell; HSC, hematopoietic stem cell; IL, interleukin; MMP, matrix-metalloproteinase; NK, natural killer; TAM, tumor-associated macrophage; TEM, Tie2-expressing macrophage; VEGF, vascular endothelial growth factor.

\footnotetext{
*Address correspondence to: Michele De Palma. Angiogenesis and TumorTargeting Research Unit, San Raffaele Scientific Institute, 20132-Milan, Italy. e-mail: depalma.michele@hsr.it
}

Final, author-corrected PDF published online: 10 June 2011.

ISSN: Online 1696-3547, Print 0214-6282 
stimulate angiogenesis and facilitate tissue remodeling by secreting a number of proteases and growth factors (Pollard, 2009). In many tissues, macrophages can be identified by their expression of a few distinguishing markers, such as F4/80 (in the mouse) and CD68, an endosomal glycoprotein also known as macrosialin (both in mouse and human).

\section{The ontogeny of macrophages}

The first macrophages appear very early during mouse development, precisely at 7.5 days post-coitum $(d p c)$. Bertrand and co-workers proposed a three-wave model for macrophage ontogeny, which takes place in the extra-embryonic yolk sac before the onset of definitive hematopoiesis (Bertrand et al., 2005). The first macrophages that are found in the yolk sac have been reported to be of maternal origin; these maternally-derived macrophages might constitute a transient population and rapidly decrease at 8.5-9 $d p c$. The second and third macrophage populations appear in the yolk sac between 8 and $9 d p c$ and are derived from monopotent and erythromyeloid macrophage precursor cells, respectively (Bertrand et al., 2005). Starting from $8.5 d p c$, macrophages can be readily identified in both extra-embryonic and embryonic tissues, including yolk sac, neural, inter-somitic and inter-digital tissues (Pucci et al., 2009). Definitive hematopoiesis (i.e., the generation of hematopoietic cells from multipotent HSCs) starts at 9.5-10 dpc in the Aorto-Gonado-Mesonephron (AGM) region of the embryo to continue in the fetal liver, spleen and BM at later stages of development. From the onset of definitive hematopoiesis throughout adult life, macrophages are thought to originate from HSCs through circulating monocyte intermediates. However, the precise origin of tissue-resident (e.g., the long-lived liver Kupffer cells and brain microglia) as well as inflammatory macrophages (i.e., macrophages that acutely infiltrate inflamed tissues) is still a matter of debate (Geissmann et al., 2003; Geissmann et al., 2010). For instance, it is not clear whether Kupffer cells and microglia originate from adult HSC-derived monocytes or self-renewing, nonHSC-derived macrophage precursors of yolk-sac origin (Chan et al., 2007; Geissmann et al., 2010). A recent study showed that postnatal HSCs do not significantly contribute to microglia homeostasis in the adult mouse brain (Ginhoux et al., 2010). In vivo lineage tracing studies indeed showed that brain microglial cells derive from primitive myeloid progenitors that arise before $8 \mathrm{dpc}$ (Ginhoux et al., 2010). There is also evidence that, during inflammation, tissue macrophages undergo rapid in situ proliferation in order to increase population density (Jenkins et al., 2011). These recent studies therefore indicate that resident macrophages can self-renew and expand in situ under the influence of appropriate stimuli and/or at selected anatomical sites (Ginhoux et al., 2010; Jenkins et al., 2011). Nevertheless, it is also clear that total body irradiation can enhance tissue-resident macrophage turnover from BM-derived, circulating monocytes (Biffi et al., 2004; De Palma et al., 2003).

\section{The role of macrophages in development}

Several transcription factors have been implicated in monocyte/ macrophage development and commitment. PU.1 regulates both embryonic and adult hematopoiesis by interacting with many other transcription factors, including GATA1. PU.1 $1^{-/-}$mice die at a late embryonic stage or shortly after birth and have impaired myelo- and Iymphopoiesis (Iwasaki and Akashi, 2007). The conditional deletion of PU.1 in the adult BM results in a complete loss of common myeloid (CMP) and lymphoid (CLP) progenitors, but not megakaryocyte-erythroid progenitors (MegE), indicating that PU.1 is necessary for multipotent progenitors to proceed to the CMP/ CLP but not MegE stage (Iwasaki and Akashi, 2007). Among the differentiated hematopoietic cells, PU. 1 is expressed in the myeloid but not lymphoid, megakaryocyte and erythroid lineages, indicating a role for PU. 1 in myeloid cell commitment and differentiation. PU.1 modulates the expression of several myeloid-specific genes, including granulocyte-colony stimulating factor (G-CSF) and granulocyte/ monocyte-colony stimulating factor (GM-CSF) receptors (Burda et al., 2010). Because PU. $1^{--}$mice display only minor erythropoietic and megakariocytopoietic defects and remain vital until perinatal developmental stages, they represent a widely used model to study the effects of macrophage deficiency on organogenesis.

PU.1-deficient mice display an osteopetrotic phenotype (Tondravi et al., 1997). Osteopetrosis is a metabolic bone disease characterized by the lack of BM cavities and defects in bone resorption. These defects are due to the lack of osteoclasts, a form of highly specialized macrophage that resides in the bone and regulates its remodeling. Similar to PU. $1^{-/-}$mice, Csf- $1^{\text {op/op }}$ mice (which lack CSF-1, a key growth factor responsible for the proliferation, differentiation, survival, and chemotaxis of monocytes/macrophages and

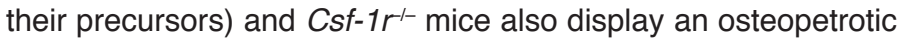
phenotype. Interestingly, both Csf-1 ${ }^{\text {op/op }}$ and $C s f-1 r^{-1-}$ mutant mice are severely depleted of most, but not all macrophage populations (Pollard, 2009). Although Csf-1 ${ }^{\text {op/op }}$ mice have many developmental abnormalities that can be attributed to the lack of individual macrophage populations (Pollard, 2009), they are viable and can be used as a model of adult macrophage deficiency in several experimental settings, including tumor progression (Lin et al., 2001). The phenotype of $C s f-1 r^{-1}$ mice is similar to that of Csf-1 op/ op mice, but the former are more severely affected and rarely live beyond a few weeks of age.

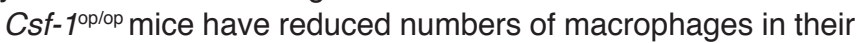
tissues, particularly in the kidney, mammary and pancreatic glands. In the developing mammary gland, CSF-1 secreted by epithelial cells recruits CSF-R $1^{+}\left(\mathrm{CD} 115^{+}\right)$macrophages to the terminal end buds (Pollard, 2009). At this anatomical site, macrophages produce many growth factors and proteases that facilitate epithelial cell growth and invasion of the surrounding connective tissue; in fact, Csf-1 ${ }^{\mathrm{op} / \mathrm{op}}$ mice show reduced complexity of mammary ductal branching due to impaired recruitment of infiltrating macrophages at the terminal end buds (Pollard, 2009).

Rae and co-workers studied embryonic macrophages in Csf$1 r$-GFP transgenic mice, which express GFP preferentially in myelomonocytic cells (Rae et al., 2007). Csf-1r-GFP-positive macrophages represented $3-10 \%$ of all embryo-derived cells at $11.5 d p c$ and were particularly abundant in developing limbs, lens, thymus and olfactory bulb. The authors then isolated GFP-positive macrophages from the brain, lung and kidney of $12 d p c$ embryos and compared their gene expression with that of macrophages isolated from adult organs. Microarray analyses indicated that embryonic macrophages, as compared to their adult macrophage counterpart, were enriched in transcripts associated with "woundhealing" and "angiogenesis" signatures. Among the up-regulated genes were mannose receptor (Mrc1), the scavenger receptors Msr1 and Mrs2, the chemokine Ccl24, and the complement protein 
C1q (Rae et al., 2007). These genes are involved in recognition and engulfment of dying cells and components of the ECM, and are also up-regulated in tumor-associated macrophages (TAMs), as discussed below.

In the kidney, organogenesis involves the infiltration of the metanephric mesenchyme by the ureteric bud and subsequent branching morphogenesis. Rae and co-workers also reported that macrophages associate with developing renal tubules and blood vessels, perhaps facilitating their invasion and expansion into the surrounding connective tissue (Rae et al., 2007). The authors further showed that embryonic macrophages promote tissue growth in metanephric explant cultures of 11.5 dpcembryos, partially in a CSF-1-dependent manner (Rae et al., 2007). These data corroborate the notion that macrophages orchestrate tissue and organ morphogenesis by producing chemokines that in turn attract and/or activate other cell types (e.g., fibroblasts) in the tissue microenvironment. Thus, the CSF-1 / CSF-1R axis - via its effects on myelo-monocytopoiesis - represents a molecular hub that is important for physiologic tissue growth and remodeling, both in development and adulthood.

\section{Macrophage regulation of angiogenesis in development and post-natal tissue remodeling}

The formation of new, functional blood vessels requires the sprouting of pre-existing blood vessels and their subsequent fusion with other blood vessels, a process known as vascular anastomosis (Ribatti, 2010; Eichmann et al., 2004; Carmeliet, 2005). Little is known about the mechanisms by which the endothelial cells (ECs) present at the leading edge of vascular sprouts (named endothelial "tip" cells) integrate directional cues from the environment and fuse to form new functional blood vessels. Fantin and co-workers have recently shown that yolk sac-derived macrophages mediate the fusion of endothelial tip cells and increase vascular complexity in the developing hindbrain (Fantin et al., 2010).

In wild-type embryos, yolk sac-derived macrophages were often observed in close association with sprouting blood vessels (Fantin et al., 2010). In the subventricular zone of the hindbrain, macrophages accumulated between 10-11.5 dpc, a time point during which blood vessels branch to form the subventricular vascular plexus. Once the subventricular vascular plexus had formed, macrophages decreased in this area while accumulated in deeper brain layers, concomitantly to the branching of radial arteries (Fantin et al., 2010). These data suggest that macrophages associate with sites of active vascular branching in the developing hindbrain. To further study the role of macrophages in vascular patterning, the authors used macrophage-deficient PU.1 $1^{-/-}$embryos (Fantin et al., 2010). They found that the hindbrain of PU.1 mutants displayed normal numbers of vascular sprouts but fewer intersections among the blood vessels, indicating reduced vascular network complexity. Of note, the vascular phenotype of PU. $1^{-/-}$embryos was less severe than that of Vegfa ${ }^{120 / 120}$ mutants, which have impaired angiogenesis due to the absence of the master proangiogenic factor, vascular endothelial growth factor (VEGF)-A. Together, these findings suggest that macrophages support blood vessel formation downstream to VEGF-induced angiogenesis (Fantin et al., 2010). The authors further showed that yolk sac-derived but not circulating monocyte-derived macrophages are important for brain angiogenesis in the developing brain. This may indicate that yolk sac macrophages infiltrate the brain before a functional circulation is established (Fantin et al., 2010), in agreement with other studies (Ginhoux et al., 2010).

From a mechanistic standpoint, the study of Fantin and co-workers suggested that macrophages promote vascular anastomosis in the developing hindbrain by physically assisting endothelial tip cell fusion through direct cell-to-cell contacts (Fantin et al., 2010). A similar mechanism has been described recently by Rymo and coworkers, who showed that macrophages/microglial cells associate with endothelial tip cells also in the developing retina (Rymo et al., 2011). Retinal macrophages were frequently found at sites where endothelial tip cells contact each other through their filopodia, and were present at 1:2 numeric ratio with the endothelial tip cells. These observations suggest that each macrophage/microglial cell can theoretically contact two neighboring endothelial tip cells. Interestingly, genetic ablation of microglia in Csf-1 ${ }^{\mathrm{op} / \mathrm{op}}$ mice determined a sparser vascular network associated with reduced numbers of filopodia-bearing sprouts. Moreover, the retinal vascular sprouts of macrophage-deficient mice were fewer and mostly radially-oriented, whereas those of wild-type mice displayed much higher complexity and were both radially- and forward-oriented, thus forming more intersections (Rymo et al., 2011). The authors then asked whether microglial cells stimulate vessel branching through direct contacts with the endothelial network. To address this question, they used mouse aortic rings cultured with or without embedded microglia, and found that microglia induced significant vascular sprouting long before the cells had made physical contact with the growing vessel network (Rymo et al., 2011). These data indicate that direct macrophage/EC cell-to-cell contacts are not strictly essential for vascular sprouting in the mouse aortic ring assay. Nevertheless, it cannot be excluded that cell-to-cell contacts are essential for directional, vascular morphogenesis in vivo, as implied by imaging data (Fantin et al., 2010; Rymo et al., 2011).

The requirement of macrophage/EC cell-to-cell contacts for vascular network formation is also suggested by studies showing that certain TAMs, namely the TIE2-expressing macrophages (TEMs), physically interact with tumor blood vessels and promote angiogenesis in mouse tumor models (De Palma et al., 2003; De Palma et al., 2005; De Palma et al., 2008; De Palma and Naldini, 2009; Pucci et al., 2009; Mazzieri et al., 2011; Welford et al., 2011; Squadrito and De Palma, 2011). Interestingly, both TEMs and embryonic macrophages that assist vascular morphogenesis express the angiopoietin receptor, TIE2 (De Palma et al., 2005; Pucci et al., 2009; Mazzieri et al., 2011; Fantin et al., 2010; Gordon et al., 2010). TIE2 is a tyrosine kinase receptor broadly expressed by ECs; it regulates angiogenesis and vascular morphogenesis both in development and adulthood (Augustin et al., 2009). Activated ECs (such as those of angiogenic blood vessels) secrete high levels of the TIE2 ligand, angiopoietin-2 (ANG2), which binds TIE2 both autocrinally on ECs and paracrinally on perivascular monocytes/ macrophages to induce them to become highly proangiogenic (Coffelt et al., 2010b). Such reciprocal signaling might regulate macrophage/EC interactions and facilitate macrophage-assisted vascular patterning both in developing organs and, as recently shown, in mouse tumor models (Mazzieri et al., 2011).

Macrophages have also been implicated in the post-natal remodeling of the retinal vasculature. Retinal development requires the formation of a temporary vascular plexus (the hyaloid vessels), which is subsequently remodeled through a vascular regression phase. 
Following remodeling, the dense primary vasculature is replaced by a narrower, smaller caliber secondary vasculature (Ishida et al., 2003; Lobov et al., 2005). In the mouse, retinal vascular regression occurs after birth and involves the activity of macrophages. Lobov and co-workers showed that the temporary hyaloid vessels can develop normally but fail to undergo regression in PU. $1^{-/-}$mice, which lack macrophages (Lobov et al., 2005). In the developing mouse retina, macrophages are the main source of WNT7B, a molecule that induces cell-cycle progression and can predispose target cells to subsequent apoptosis (Lobov et al., 2005). Rao and co-workers further showed that macrophage-mediated vascular regression involves two distinct signals, WNT7B and ANG2 (Rao et al., 2007). ANG2 has long been regarded as a natural antagonist for ANG1 (Augustin et al., 2009), although recent studies have indicated that it may also function as a context-dependent TIE2 agonist (Daly et al., 2006; Coffelt et al., 2010b). In the presence of ANG2 produced by the hyaloid blood vessels, resident macrophages upregulate WNT7B, which induces the proliferation of vascular ECs and sensitizes them to ANG2-induced apoptosis. In the absence of ANG2, ANG1 signaling prevails and EC death is suppressed (Rao et al., 2007). Together, these data suggest that macrophages directly regulate retinal vascular remodeling, but not initiation of retinal angiogenesis. Because a sizable fraction of the embryonic macrophages express the TIE2 receptor or a Tie2-GFP reporter gene in transgenic mice (Fantin et al., 2010; Pucci et al., 2009; Gordon et al., 2010), it can be envisioned that ANG2 directly acts on macrophages via TIE2 to regulate WNT7B secretion (Rao et al., 2007). It should be noted, however, that one study suggested that cytotoxic T cells - rather than macrophages - promote vascular regression and remodeling of the primary retinal plexus by inducing Fas ligand-mediated EC apoptosis (Ishida et al., 2003).

During pregnancy, angiogenesis and vascular remodeling at the maternal-fetal interface ensure continuous blood flow to the fetus. Inadequate angiogenesis and vascular remodeling limit extraembryonic trophoblast invasion of the decidua (the maternal uterine mucosa during pregnancy) and may cause pre-eclampsia and miscarriage (Harris, 2010). Both in mice and humans, macrophages and natural killer (NK) cells infiltrate the human decidua and regulate spiral artery remodeling at the maternal-fetal interface. Decidual NK cells, but not peripheral blood-derived NK cell subsets, were shown to promote trophoblast invasion and angiogenesis by secreting interleukin-8 (IL-8) and many other proangiogenic factors (Hanna et al., 2006). Interestingly, both macrophages and NK cells are absent from intact arteries, but accumulate in significant numbers in and around remodeling arteries (Harris, 2010). According to Hazan and co-workers, spiral artery remodeling may depend on matrix-metalloproteinase (MMP)-9, which is essentially produced by the macrophages and NK cells in the decidua (Hazan et al., 2010). These MMP9-expressing leukocytes were shown to be in close association with blood vessels during the early phases of remodeling, and MMP2/9 inhibition in human placental-decidual explants resulted in failure of vascular remodeling (Hazan et al., 2010). These data therefore suggest that macrophage-derived MMPs facilitate cell migration, invasion and vascular remodeling during pregnancy.

In summary, there is now compelling evidence for macrophages to regulate vascular morphogenesis during development and postnatal tissue/organ remodeling. In addition to expressing classic proangiogenic and tissue-remodeling factors, which may initiate angiogenesis, macrophages appear to support the formation of a functional vascular system by (i) assisting directional vessel growth via cell-to-cell contacts and/or their production of guidance factors that act iuxtacrinally on vascular sprouts after the induction of EC proliferation and angiogenesis; (ii) pruning primitive blood vessels (via secretion of proapoptotic factors) to remodel the vascular network.

\section{Macrophage regulation of angiogenesis in tissue injury and repair}

Macrophages likely represent the preeminent cells in the body endowed with the ability to migrate within tissues - even in hypoxic conditions - and with the capacity to modify the ECM and amplify paracrine signals. For example, in a transgenic model of ischemic cardiomyopathy in which monocytes are attracted to the myocardium by the targeted overexpression of CCL2 (a CC-chemokine formerly known as monocyte chemoattractant protein-1, MCP-1), monocytes/macrophages were found to drill tunnels in the ECM to facilitate the subsequent in-growth of new capillaries (Moldovan et al., 2000). Macrophages may thus provide temporary scaffolds or paracrine support for the expansion and maturation of vascular networks, both in development (see above) and in pathophysiological conditions.

Wound healing is a complex biological process that occurs frequently during adulthood and requires the interplay among different cell types, including keratinocytes, fibroblasts and immune cells (Martin, 1997; Rodero et al., 2011). Early studies suggested that macrophages and neutrophils play a crucial role during skin wound healing (Leibovich and Ross, 1975). Macrophages are generally believed to orchestrate the repair process by acting both as phagocytes (to clear cellular debris) and as a major source of growth factors (e.g., proangiogenic factors; epithelial-cell growth factors; etc...). Martin and co-workers however showed that macrophage-deficient PU. $1^{-/}$mice are able to repair skin wounds with similar time course to wild-type siblings (Martin et al., 2003). Yet, healed skin wounds in macrophage-deficient mice showed minimal scar formation, a likely consequence of the reduced inflammatory response and lower rate of keratinocyte cell death occurring in the absence of proinflammatory macrophages (Martin et al., 2003). More recent studies however showed that macrophages recruited during the diverse phases of skin repair (i.e., the inflammatory; tissue formation; and tissue maturation phases) exert distinct functions (Lucas et al., 2010). By crossing transgenic mice that express the CRE recombinase from the lysozyme $M($ LysM) promoter, with transgenic mice that express the human diphtheria toxin (DT) receptor upon CRE-mediated recombination, the authors generated mice in which depletion of macrophages and other myeloid cells can be induced during specific stages of the repair response. Depletion of myeloid cells during the early stages of the repair response (inflammatory phase) significantly reduced the formation of vascularized granulation tissue, impaired epithelialization, and resulted in minimized scar formation (Lucas et al., 2010), the latter finding in agreement with the study of Martin and co-workers (Martin et al., 2003). In contrast, depletion of macrophages during the subsequent phase of the repair response (tissue formation) caused severe hemorrhage in the wound tissue and prevented tissue maturation and wound closure. Finally, macrophage depletion during the late stage of repair (tissue maturation) did not significantly impact the outcome of the 
repair response (Lucas et al., 2010). These interesting observations suggest that macrophages exert distinct functions during the diverse phases of skin repair. In particular, macrophages appear to play an important role in promoting (i) angiogenesis in the granulation tissue during the early phase of skin repair; (ii) vascular maturation and stabilization in the subsequent phases (Lucas et al., 2010).

Tissue macrophages can display different activation states according to the stimuli present in the local microenvironment. In several microbial infections and other inflammatory conditions, the products of activated T helper-1 (Th1) lymphocytes and NK cells (e.g., interferon-gamma, IFN $\gamma$ ) can induce macrophages to undergo a "classical activation" (or "M1") program that enhances their cytotoxic and antimicrobial activity. Conversely, signals produced by Th2 polarized lymphocytes (e.g., IL4, IL10 or IL13) can induce macrophages to acquire an "alternative activation" (or "M2") status in various pathophysiological conditions, such as wound healing and granuloma formation, or in certain disease conditions, such as fibrosis, atherosclerosis, stroke, and cancer (Gordon, 2003; Gordon and Martinez, 2010; Mantovani et al., 2002).

Distinct "types" of wound healing macrophages have been described to date; their phenotypic and functional characterization is, however, not yet exhaustive (Rodero et al., 2011). Okuno and co-workers recently showed that the majority of BM-derived cells infiltrating both acute (dorsal excisional ear punch) and chronic (decubitus ulcer) skin wounds are macrophages (Okuno et al., 2011). Most of these macrophages display an "alternatively" activated or M2-like phenotype (Gordon, 2003; Gordon and Martinez, 2010), e.g., express high levels of MRC1 and low levels of nitric oxide synthase-2 (NOS2) and IL-6. Interestingly, the authors found that the majority of these macrophages were located in perivascular areas at the wound healing site (Okuno et al., 2011).

Recruitment of macrophages to the wound healing site may rely on different signals, such as CCL2/MCP1, macrophage inflammatory protein-1alpha (MIP1 $\alpha$ ), but also stromal cell-derived factor 1 (SDF1)/CXCL12 and G-CSF (Wu et al., 2010). Okuno and co-workers showed that CSF-1 is early up-regulated at the wound healing site and plays an important role in recruiting macrophages (Okuno et al., 2011). This was confirmed by inhibition of CSF-1R or CSF-1, which resulted in $80 \%$ reduction of the macrophage infiltrate. Moreover, the authors observed delayed wound healing

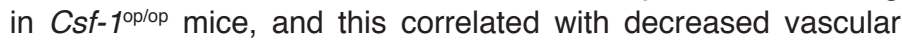
density. However, the overall amounts of classic proangiogenic factors produced at the wounded site did not differ between Csf-1 op/ op and wild-type mice, whereas MMPs were significantly reduced. Thus, wound healing macrophages may exert their proangiogenic programs by physically interacting with sprouting blood vessels, facilitating their growth by degrading the ECM, and/or promoting vascular remodeling via non-canonical (e.g., VEGF-independent) angiogenic pathways (Okuno et al., 2011).

Sindrilaru and co-workers recently reported that human chronic venous ulcers contain increased proportions of macrophages expressing M1-type markers (e.g., NOS2 and tumor necrosis factor-alpha $(\mathrm{TNF} \alpha)$ ) than healing wounds (Sindrilaru et al., 2011). As mentioned above, "classically" activated or M1-polarized macrophages sustain tissue inflammation and actively phagocytose dead cells, cellular debris and microbes (Gordon, 2003; Gordon and Martinez, 2010). The authors found that iron deposits and their subsequent uptake by macrophages in chronic wounds impaired macrophage switching from the M1-like to the M2-like phenotype, a phenotypic switch that instead occurs in healing wounds (Sindrilaru et al., 2011). Together with previous reports (Okuno et al., 2011; Rodero et al., 2011), this study suggests that M1-like macrophages prevail in early or chronic wounds, where they prevent infection and help clearance of necrotic tissue (Sindrilaru et al., 2011). Conversely, proficient wound healing requires the participation of M2-like macrophages, which may either originate from M1-like macrophages or from different monocyte precursors.

Macrophages have also been implicated in the healing/regeneration of the nervous tissue. Barrette and co-workers showed that the depletion of CD11 $\mathrm{b}^{+}$myeloid cells in CD11b-HSV-thymidine kinase transgenic mice impaired recovery of sciatic nerve function after injury (Barrette et al., 2008). Depletion of myeloid cells was associated with a significant decrease in vascular density and delayed neural cell proliferation (Barrette et al., 2008). Kigerl and co-workers further observed that M2-like macrophages transiently increase at the spinal cord injury site (Kigerl et al., 2009). In this study, the authors also showed that neurons co-cultured with M2-like macrophages displayed more neuritis, and their axons projected over longer distances, compared to neurons co-cultured with M1-like macrophages (Kigerl et al., 2010). These interesting observations suggest that M2-like macrophages participate in both vascular network formation and neural development. In this regard, it is increasingly appreciated that blood vessels and neurons share cell-surface receptors and guidance cues, such as several members of the VEGF and Semaphorin/Plexin families, to control their behavior during embryogenesis and, possibly, also in selected pathophysiological conditions during post-natal life (Fantin et al., 2009).

\section{The role of macrophages in lymphangiogenesis}

Lymphatic vessels regulate tissue fluid homeostasis and play a crucial role in immune cell trafficking. The molecular pathways involved in lymphangiogenesis have been elucidated more in detail in the context of development. In the adult, lymphangiogenesis occurs in inflammation, tumors, and during the process of wound healing. In the mouse embryo, lymphangiogenesis begins at 9.5$10.5 \mathrm{dpc}$, after the cardiovascular system is formed (Tammela and Alitalo, 2010). Lymphatic ECs arise from specialized subpopulations of ECs in the anterior cardinal vein; these specialized ECs are positive for prospero-related homeodomain transcription factor (PROX-1), which is the master molecular switch for lymphatic EC determination, and hyaluronan receptor-1 (LYVE1).

In addition to angiogenesis, macrophages also appear to regulate lymphangiogenesis. For example, CSF-1 deficiency in Csf-1op/op mice reduces the abundance of macrophages in the developing trachea, resulting in defects in both vascular and lymphatic development (Kubota et al., 2009). Bohmer and co-workers recently described a population of monocyte-lineage cells, defined by the expression of Syk tyrosine kinase (Bohmer et al., 2010). These cells were characterized by their robust expression of proangiogenic factors, such as fibroblast growth factor-2 (FGF2), platelet-derived growth factor-B (PDGF-B), MMP2 and MMP9, as well as lymphangiogenic growth factors (VEGF-C and VEGF-D). Interestingly, the skin of Syk-decifient embryos showed increased accumulation of these proangiogenic monocyte-lineage cells, which expressed a more pronounced proangiogenic profile than their wild-type counterpart (Bohmer et al., 2010). The enhanced infiltration by 
lymphangiogenic monocytes in Syk-deficient embryos caused lymphatic hyperplasia, vessel dilation leading to blood-lymphatic shunts, and lethality during mid-gestation. Interestingly, the Sykexpressing monocytes also express TIE2 (Bohmer et al., 2010) and display many similarities with the TIE2-expressing monocytes/ macrophages found in tumors and embryos (Pucci et al., 2009). Another recent study showed that clodronate-liposome-mediated depletion of VEGF-C-expressing, dermal macrophages counteracts hyperplasia of lymphatic capillaries in response to high-salt diet (Machnik et al., 2009). Taken together, these findings (Bohmer et al., 2010; Machnik et al., 2009) indicate that, in addition to angiogenesis, monocyte/macrophages may also regulate lymphangiogenesis. Furthermore, they suggest that the enhanced recruitment of monocyte/macrophages that occurs in certain pathological conditions (e.g., salt-sensitive hypertension) may induce lymphatic hyperplasia and pathological lymphangiogenesis.

Another recent report implicated macrophages in lymphangiogenesis (Gordon et al., 2010). In this study, the authors showed that 14.5-18.5 dpc PU. $1^{-1-}$ and Csf-1r $r^{-1}$ mice display hyperplastic lymphatic vessels in the skin. This phenotype appears to be due to the specific lack of myeloid-lineage cells, since lymphocyte-deficient Rag2 $^{--}$mice did not show hyperplastic lymphatic vessels in the skin (Gordon et al., 2010). Although skin macrophages appeared dispensable for lymphatic vessel sprouting and branching, they influenced lymphatic vessel caliber, possibly by controlling either the proliferation or apoptosis of lymphatic ECs in the developing skin. In apparent contradiction with other studies (Bohmer et al., 2010), the findings of Gordon and co-workers (Gordon et al., 2010) also imply that macrophages may not comprise the principal source of pro-lymphangiogenic growth factors (including VEGF-C and VEGFD) in the embryonic dermal microenvironment, and also suggest that the sources of proliferative and patterning signals driving embryonic lymphangiogenesis are likely to be distinct (Gordon et al., 2010). The authors further characterized the dermal macrophages and identified a subset of LYVE1 + cells that intimately associated with the developing lymphatic vasculature. Interestingly, the gene expression profile of these LYVE1+ macrophagesoverlaps with that of both embryonic and tumor-derived TEMs (Pucci et al., 2009). Because these LYVE1+ macrophages provide only $10 \%$ of the prolymphangiogenic molecules present in the dermal microenvironment (Gordon et al., 2010), it is tempting to speculate that, in analogy with developmental (Fantin et al., 2010) and tumor (Mazzieri et al., 2011) angiogenesis, TEM-like embryonic macrophages are not essential for lymphatic vessel sprouting but play an important role in lymphatic vessel expansion and remodeling (Gordon et al., 2010).

\section{Similarities between embryonic and tumor-associated macrophages (TAMs)}

In order to grow over a few millimeters in size, incipient tumors need to generate their own vasculature to enable oxygen and nourishment delivery to the expanding tumor cells (Carmeliet, 2005). It is now well established that tumor-associated stromal cells such as macrophages (TAMs), neutrophils and fibroblasts cooperate with tumor cells to facilitate angiogenesis in tumors (Squadrito and De Palma, 2011; Coffelt et al., 2010a; Egeblad et al., 2010; Murdoch et al., 2008).

In several mouse tumor models, macrophages represent the most abundant tumor-infiltrating myeloid cells (Squadrito and De Palma, 2011). The early finding that high macrophage counts correlate with higher tumor angiogenesis in some human tumor types (Leek et al., 1996) suggested that these cells might exert a direct proangiogenic function in tumors. However, compelling evidence for the ability of macrophages to promote tumor angiogenesis was only provided using mouse models of in vivomonocyte/macrophage deficiency or adoptive transfer. De Palma and co-workers showed that the genetic depletion of TEMs was sufficient to robustly inhibit angiogenesis in various subcutaneous tumor models (De Palma et al., 2003; De Palma et al., 2005). Moreover, by crossing Csf1op/ op mice with mice that develop oncogene-induced mammary
A

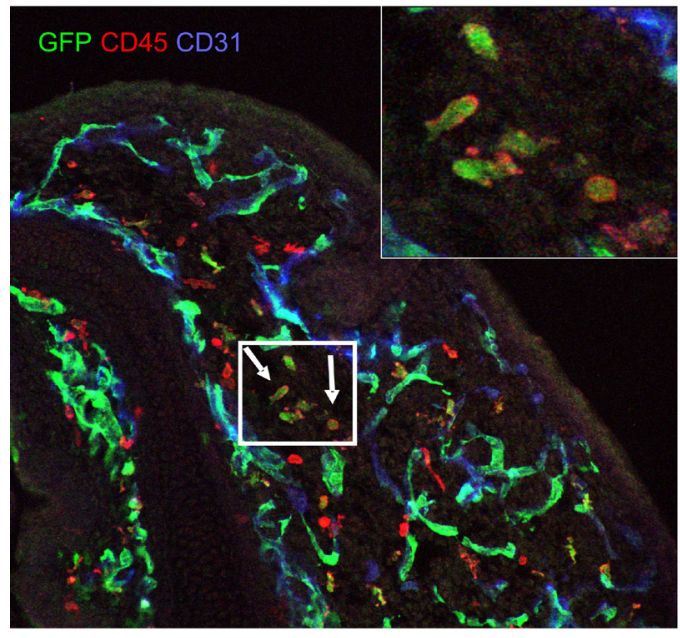

B
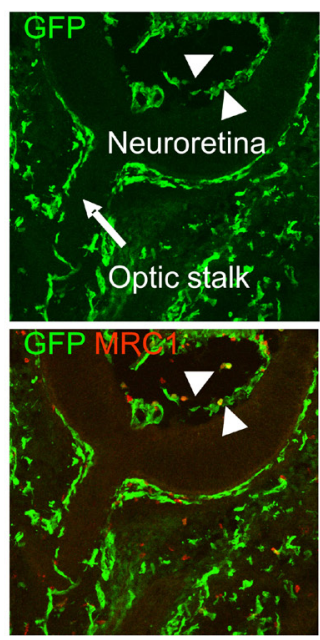
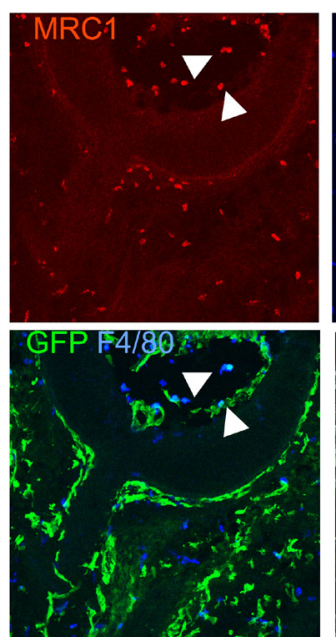

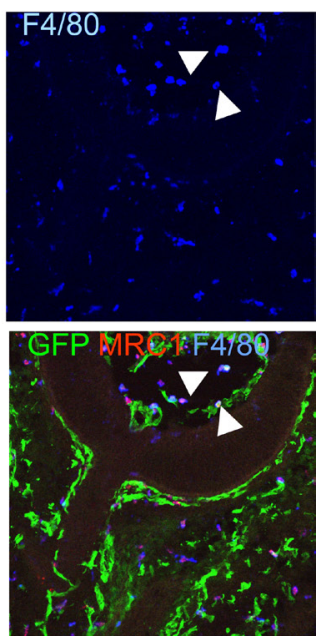

Fig. 1. Presence of Tie2-GFP+ macrophages in the mouse embryo. (A) SeveralTie2-GFP+CD45+ Tie2-expressing macrophages (TEMs; white arrows and inset on top right) are present in the developing brain of a Tie2-GFP transgenic embryo. Note that in Tie2-GFP transgenic mice, both vascular endothelial cells and TEMs express GFP from the Tie2 promoter/enhancer. TEMs are labeled in green (GFP); hematopoietic cells in red (CD45); blood vessels in blue (CD31). (B) Tie2-GFP+F4/80+MRC1+ TEMs (arrowheads) are present in the developing eye of a Tie2-GFP transgenic embryo. Note the proximity of GFP+ TEMs to the GFP+ hyaloids blood vessels. TEMs are labeled in green (GFP); macrophages in red (MRC1) and blue (F4/80). Sections were obtained from late-stage embryos. 
tumors (MMTV-PyMT), Lin and co-workers found that the tumors of these macrophage-deficient mice had reduced angiogenesis and delayed progression to malignancy (Lin et al., 2006). Finally, the depletion of tissue macrophages by clodronate-liposomes resulted in delayed angiogenesis and tumor growth in various tumor models (Zeisberger et al., 2006). Together, these studies have highlighted the important role played by macrophages in tumor angiogenesis. Several mechanisms may be involved, such as macrophage production of a plethora of proangiogenic growth factors and chemokines. Moreover, TAMs can mobilize angiogenic growth factors embedded in the ECM thanks to their secretion of many proteases and other matrix-remodeling enzymes (Murdoch et al., 2008). As mentioned above, similar to embryonic macrophages (Fantin et al., 2010), subsets of TAMs - such as TEMs - can also interact directly with tumor ECs to facilitate blood vessel growth (De Palma et al., 2005; Mazzieri et al., 2011).

Ojalvo and co-workers performed gene expression analyses of TAMs isolated from late-stage, MMTV-PyMT mammary tumors, and compared their gene expression signature with that of splenic and embryonic macrophages (Ojalvo et al., 2009). The data highlighted the tissue trophic functions of TAMs and their similarity with embryonic but not splenic macrophages. Pucciand co-workers further showed that TIE2 ${ }^{+}$embryonic/fetal macrophages, which appear at $8.5 \mathrm{dpc}$, show common features with tumor-infiltrating TEMs (Pucci et al., 2009). In the embryo, TEMs are particularly enriched in the mesenchyme surrounding neural organs; these TIE2+ macrophages are often found in close proximity to blood vessels at distinct anatomical sites, including the hyaloid cavity of the eye (Fig. 1). Gene expression analyses further indicated that embryonic/fetal TEMs are molecularly similar to tumor-associated TEMs (Pucci et al., 2009). Indeed, embryonic TEMs express the typical tumor TEM markers MRC1, haptoglobin/hemoglobin scavenger receptor (CD163), neuropilin-1 (NP1), stabilin-1 (STAB1), and LYVE1. Interestingly, tumor TEMs express lower VEGF levels that classic, TIE2-negative (inflammatory) TAMs (Pucci etal., 2009). This may be consistent with the observation that TEMs preferentially reside around newly formed tumor blood vessels in viable tumor areas (note that VEGF is strongly upregulated in cells that reside in hypoxic or necrotic microenvironments). The finding of lower VEGF expression in TEMs than classic TAMs also suggests that the former are likely to promote tumor angiogenesis mostly in a VEGF-independent manner. Our recent studies suggest that tumor TEMs, similar to TIE2+ embryonic macrophages (Fantin et al., 2010), promote the formation of a functional tumor-associated vasculature by associating with sprouting tumor blood vessels, and that this function is regulated by the ANG2/TIE2 axis in macrophages (Mazzieri et al., 2011).

It should be emphasized that embryonic and tumor macrophages have different origins: whereas fetal macrophages are derived from the extra-embryonic mesoderm (yolksac), tumor macrophages likely originate from HSC-derived monocytes (De Palma et al., 2003). Thus, it can be speculated that embryonic macrophages and specific TAM subsets, such as TEMs, express similar gene signatures as a result of convergent functional adaptation. Whereas TEMs generally constitute a minor macrophage subset in mouse tumors (Squadrito and De Palma, 2011), the majority of macrophages seem to express a TEM-like phenotype in the developing embryo (Fantin et al., 2010; Pucci et al., 2009). This may reflect the fact that variably inflamed, hypoxic, necrotic, hemorrhagic, as well as avascular or highly vascularized microenvironments are present in any given tumor. Such degree of histological heterogeneity is accompanied by a remarkable heterogeneity of macrophage phenotypes (Lewis and Pollard, 2006; Mantovani et al., 2008; Qian and Pollard, 2010; Squadrito and De Palma, 2011). It appears that tumor TEMs mostly reside in viable and angiogenic tumor areas, whereas they are mostly excluded from necrotic, inner tumor areas (De Palma and Naldini, 2009). Developing embryos do not contain inflamed, necrotic or hemorrhagic microenvironments, but require sustained angiogenesis and tissue remodeling to support organ growth. These microenvironmental features may skew macrophage recruitment/differentiation toward a TEM-like (proangiogenic and pro-remodeling) phenotype, and even prevent the generation of cytotoxic (or proinflammatory) macrophages.

A recent study reported the generation of TEM-like, proangiogenic monocytes/macrophages from human embryonic stem cells (ESCs) (Klimchenko et al., 2011). These TIE2+ ESC-derived monocytic cells displayed enhanced expression of genes encoding matrix-degrading enzymes, proangiogenic factors and scavenger receptors, as was previously reported for tumor TEMs and other M2-like macrophages (Pucci et al., 2009). Furthermore, they promoted vascular remodeling in xenotransplanted human tumors (Klimchenko et al., 2011). The generation of TEM-like cells from ESCs may offer a valuable experimental tool for in vitro studies and, possibly, provide a source of proangiogenic cells for the treatment of ischemia, injured tissues and chronic wounds. Finally, it is conceivable that better characterizing the developmental roles of macrophages - including their genetic programs facilitating angiogenesis and tissue morphogenesis in developing organs - may provide clues to TAM function in cancer and identify molecular targets that could be exploited to inhibit their proangiogenic and protumoral activities in human neoplasia and in other diseases.

\section{Acknowledgements}

The authors thank all the members of the Angiogenesis and Tumor Targeting Unit, and apologize to all the authors whose work could not be cited due to space limitations. MDP acknowledges grant support from the European Research Council (Starting Grant 243128/TIE2+Monocytes), Associazione Italiana per la Ricerca sul Cancro (IG-2010) and Fondazione Berlucchi.

\section{References}

AUFFRAY C, FOGG D, GARFA M, ELAIN G, JOIN-LAMBERT O, KAYAL S, SARNACKI S, CUMANO A, LAUVAU G, GEISSMANN F. (2007). Monitoring of blood vessels and tissues by a population of monocytes with patrolling behavior. Science 317: 666-670.

AUGUSTIN HG, YOUNG KOH G, THURSTON G, ALITALO K. (2009). Control o vascular morphogenesis and homeostasis through the angiopoietin-Tie system. Nat Rev Mol Cell Biol 10: 165-177.

BARRETTE B, HÉBERT MA, FILALI M, LAFORTUNE K, VALLIĖRES N, GOWING G, JULIEN JP, LACROIX S. Requirement of myeloid cells for axon regeneration. (2008) J Neurosci. 28: 9363-9376.

BERTRAND JY, JALIL A, KLAINE M, JUNG S, CUMANO A, GODIN I. (2005) Three pathways to mature macrophages in the early mouse yolk sac. Blood 106: 3004-3011.

BIFFI A, DE PALMA M, QUATTRINI A, DEL CARRO U, AMADIO S, VISIGALLI I, SESSA M, FASANO S, BRAMBILLA R, MARCHESINI S et al., (2004). Correction of metachromatic leukodystrophy in the mouse model by transplantation of genetically modified hematopoietic stem cells. J Clin Invest 113: 1118-1129.

BOHMER R, NEUHAUS B, BUHREN S, ZHANG D, STEHLING M, BOCK B, KIEFER F. (2010) Regulation of developmental lymphangiogenesis by Syk(+) leukocytes. 
Dev Cell 18: 437-449.

BURDA P, LASLO P, STOPKA T. (2010) The role of PU.1 and GATA-1 transcription factors during normal and leukemogenic hematopoiesis. Leukemia24:1249-1257.

CARMELIETP. (2005) Angiogenesis in life, disease and medicine. Nature438:932-936.

CHAN WY, KOHSAKA S, REZAIE P. (2007). The origin and cell lineage of microglia: new concepts. Brain Res Rev 53: 344-354.

COFFELT SB, LEWIS CE, NALDINI L, BROWN JM, FERRARA N, DE PALMA M. (2010a) Elusive identities and overlapping phenotypes of proangiogenic myeloid cells in tumors. Am J Pathol 176:1564-1576.

COFFELT SB, TAL AO, SCHOLZ A, DE PALMA M, PATEL S, URBICH C, BISWAS SK, MURDOCH C, PLATE KH, REISS Y et al., (2010b) Angiopoietin-2 Regulates Gene Expression in TIE2-Expressing Monocytes and Augments Their Inherent Proangiogenic Functions. Cancer Res. 70: 5270-5280

DALY C, PASNIKOWSKI E, BUROVA E, WONG V, ALDRICH TH, GRIFFITHS J, IOFFE E, DALY TJ, FANDL JP, PAPADOPOULOS N et al., (2006). Angiopoietin-2 functions as an autocrine protective factor in stressed endothelial cells. Proc Natl Acad Sci USA 103: 15491-15496.

DE PALMA M, MAZZIERI R, POLITI LS, PUCCI F, ZONARI E, SITIA G, MAZZOLENI S, MOI D, VENNERI MA, INDRACCOLO S et al., (2008). Tumor-targeted interferon-alpha delivery by Tie2-expressing monocytes inhibits tumor growth and metastasis. Cancer Cell 14: 299-311.

DE PALMA M, NALDINI L. (2009). Tie2-expressing monocytes (TEMs): Novel targets and vehicles of anticancer therapy? Biochim Biophys Acta 1796: 5-10.

DE PALMA M, VENNERI MA, GALLI R, SERGI SERGI L, POLITI LS, SAMPAOLES I M, NALDINI L. (2005). Tie2 identifies a hematopoietic lineage of proangiogenic monocytes required for tumor vessel formation and a mesenchymal population of pericyte progenitors. Cancer Cell 8: 211-226.

DE PALMA M, VENNERI MA, ROCA C, NALDINI L. (2003). Targeting exogenous genes to tumor angiogenesis by transplantation of genetically modified hematopoietic stem cells. Nat Med 9: 789-795.

EGEBLAD M, WERB Z. (2002). New functions for the matrix metalloproteinases in cancer progression. Nat Rev Cancer 2: 161-174.

EGEBLAD M, NAKASONE ES, WERB Z. (2010) Tumors as organs: complex tissues that interface with the entire organism. Dev Cell. 18: 884-901

EICHMANN A, YUAN L, MOYON D, LENOBLE F, PARDANAUD L, BREANT C. (2005) Vascular development: from precursor cells to branched arterial and venous networks. Int J Dev Biol. 49: 259-267.

FANTIN A, MADEN CH, RUHRBERG C. (2009) Neuropilin ligands in vascular and neuronal patterning. Biochem Soc Trans. 37: 1228-1232

FANTIN A, VIEIRA JM, GESTRI G, DENTI L, SCHWARZ Q, PRYKHOZHIJ S, PERI F, WILSON SW, RUHRBERG C. (2010) Tissue macrophages act as cellular chaperones for vascular anastomosis downstream of VEGF-mediated endothelial tip cell induction. Blood. 116: 829-840

GEISSMANN F, JUNG S, LITTMAN DR. (2003). Blood monocytes consist of two principal subsets with distinct migratory properties. Immunity 19: 71-82.

GEISSMANN F, MANZ MG, JUNG S, SIEWEKE MH, MERAD M, LEY K. (2010) Development of monocytes, macrophages, and dendritic cells. Science 327:656-661.

GINHOUX F, GRETER M, LEBOEUF M, NANDI S, SEE P, GOKHAN S, MEHLER MF, CONWAY SJ, NG LG, STANLEY ER et al., (2010) Fate mapping analysis reveals that adult microglia derive from primitive macrophages. Science 330: 841-845.

GORDON EJ, RAO S, POLLARD JW, NUTT SL, LANG RA, HARVEY NL. (2010) Macrophages define dermal lymphatic vessel calibre during development by regulating lymphatic endothelial cell proliferation. Development 137: 3899-3910.

GORDON S. (2003) Alternative activation of macrophages. Nat Rev Immunol. 3: 23-35.

GORDON S, MARTINEZ FO. (2010) Alternative activation of macrophages: mechanism and functions. Immunity. 32: 593-604

HANNA J, GOLDMAN-WOHLD, HAMANI Y, AVRAHAM I, GREENFIELD C, NATANSON-YARON S, PRUS D, COHEN-DANIEL L, ARNON TI, MANASTER I et al., (2006). Decidual NK cells regulate key developmental processes at the human fetal-maternal interface. Nat Med 12:1065-1074

HARRIS LK. (2010) Review: Trophoblast-vascular cell interactions in early pregnancy: how to remodel a vessel. Placenta 31 Suppl,S93-98.

HAZAN AD, SMITH SD, JONES RL, WHITTLE W, LYE SJ, DUNK CE. (2010) Vascular-Leukocyte Interactions. Mechanisms of Human Decidual Spiral Artery Remodeling in Vitro. Am J Pathol 177: 1017-1030.
ISHIDA S, YAMASHIRO K, USUI T, KAJI Y, OGURA Y, HIDA T, HONDA Y, OGUCHI Y, ADAMIS AP. (2003). Leukocytes mediate retinal vascular remodeling during development and vaso-obliteration in disease. Nat Med 9: 781-788.

IWASAKI H, AKASHI K. (2007). Myeloid lineage commitment from the hematopoietic stem cell. Immunity 26: 726-740.

JAIN RK. (2005). Normalization of tumor vasculature: an emerging concept in antiangiogenic therapy. Science 307:58-62.

JENKINS SJ, RUCKERLD, COOK PC, JONES LH, FINKELMAN FD, VAN ROOIJEN N, MACDONALD AS, ALLEN JE. (2011) Local Macrophage Proliferation, Rather than Recruitment from the Blood, Is a Signature of TH2 Inflammation. Science DOI: $10.1126 /$ science.1204351

KIGERL KA, GENSEL JC, ANKENY DP, ALEXANDER JK, DONNELLY DJ, POPOVICH PG. (2009) Identification of two distinct macrophage subsets with divergent effects causing either neurotoxicity or regeneration in the injured mouse spinal cord. J Neurosci. 29:13435-13444.

KLIMCHENKO O, DI STEFANO A, GEOERGER B, HAMIDI S, OPOLON P, ROBERT T, ROUTHIER M, EL-BENNA J, DELEZOIDE AL, BOUKOUR S, LESCURE B, SOLARY E, VAINCHENKER W, NOROL F. (2011) Monocytic cells derived from human embryonic stem cells and fetal liver share common differentiation pathways and homeostatic functions. Blood 117:3065-3075.

KUBOTA Y, TAKUBO K, SHIMIZU T, OHNO H, KISHI K, SHIBUYA M, SAYA H, SUDA T. (2009). M-CSF inhibition selectively targets pathological angiogenesis and lymphangiogenesis. J Exp Med 206: 1089-1102.

LEEK RD, LEWIS CE, WHITEHOUSE R, GREENALL M, CLARKE J, HARRIS AL. (1996) Association of macrophage infiltration with angiogenesis and prognosis in invasive breast carcinoma. Cancer Res. 56:4625-4629

LEIBOVICH SJ, ROSS R. (1975) The role of the macrophage in wound repair. A study with hydrocortisone and antimacrophage serum. Am J Pathol. 78:71-100.

LEWIS CE, POLLARD JW. (2006). Distinct role of macrophages in different tumor microenvironments. Cancer Res 66: 605-612.

LIN EY, LI JF, GNATOVSKIY L, DENG Y, ZHU L, GRZESIK DA, QIAN H, XUE XN, POLLARD JW. (2006). Macrophages regulate the angiogenic switch in a mouse model of breast cancer. Cancer Res 66:11238-11246.

LIN EY, NGUYEN AV, RUSSELL RG, POLLARD JW. (2001). Colony-stimulating factor 1 promotes progression of mammary tumors to malignancy. $J$ Exp Med 193: $727-740$

LOBOV IB, RAOS, CARROLLTJ, VALLANCE JE, ITOM, ONDR JK, KURUPS, GLASS DA, PATEL MS, SHU W et al., (2005). WNT7b mediates macrophage-induced programmed cell death in patterning of the vasculature. Nature 437: 417-421.

LUCAS T, WAISMAN A, RANJAN R, ROES J, KRIEG T, MÜLLER W, ROERS A, EMING SA. (2010) Differential roles of macrophages in diverse phases of skin repair. J Immunol. 184:3964-3977

MACHNIK A, NEUHOFER W, JANTSCH J, DAHLMANN A, TAMMELA T, MACHURA K, PARK JK, BECK FX, MULLER DN, DERER W et al., (2009). Macrophages regulate salt-dependent volume and blood pressure by a vascular endothelial growth factor-C-dependent buffering mechanism. Nat Med 15: 545-552.

MANTOVANI A, SOZZANI S, LOCATI M, ALLAVENA P, SICAA. (2002) Macrophage polarization: tumor-associated macrophages as a paradigm for polarized M2 mononuclear phagocytes. Trends Immunol. 23:549-555.

MANTOVANI A, ALLAVENA P, SICA A, BALKWILL F. (2008). Cancer-related inflammation. Nature 454: 436-444.

MARTIN P. (1997) Wound healing--aiming for perfect skin regeneration. Science 276:75-81

MARTIN P, D'SOUZAD, MARTIN J, GROSE R, COOPER L, MAKI R, MCKERCHER SR. (2003) Wound healing in the PU.1 null mouse--tissue repair is not dependent on inflammatory cells. Curr Biol 13:1122-1128.

MAZZIERI R, PUCCI F, MOI D, ZONARI E, RANGHETTI A, BERTI A, POLITI LS, GENTNER B, BROWN JL, NALDINI L, DE PALMAM. (2011) Targeting the ANG2/ TIE2 Axis Inhibits Tumor Growth and Metastasis by Impairing Angiogenesis and Disabling Rebounds of Proangiogenic Myeloid Cells. Cancer Cell 19: 512-526.

MOLDOVANNI, GOLDSCHMIDT-CLERMONTPJ, PARKER-THORNBURG J, SHAPIRO SD, KOLATTUKUDY PE. (2000). Contribution of monocytes/macrophages to compensatory neovascularization: the drilling of metalloelastase-positive tunnels in ischemic myocardium. Circ Res 87: 378-384.

MURDOCH C, MUTHANA M, COFFELT SB, LEWIS CE. (2008). The role of myeloid cells in the promotion of tumour angiogenesis. Nat Rev Cancer 8: 618-631. 
NAHRENDORF M, SWIRSKI FK, AIKAWA E, STANGENBERG L, WURDINGER T, FIGUEIREDO JL, LIBBY P, WEISSLEDER R, PITTET MJ. (2007). The healing myocardium sequentially mobilizes two monocyte subsets with divergent and complementary functions. J Exp Med 204: 3037-3047.

OJALVO LS, KING W, COX D, POLLARD JW. (2009). High-density gene expression analysis of tumor-associated macrophages from mouse mammary tumors. Am J Pathol 174: 1048-1064.

OKUNO Y, NAKAMURA-ISHIZU A, KISHI K, SUDA T, KUBOTA Y. (2011) Bone marrow-derived cells serve as proangiogenic macrophages but not endothelial cells in wound healing. Blood 117: 5264-5272.

POLLARD JW. (2009). Trophic macrophages in development and disease. Nat Rev Immunol 9: 259-270.

PUCCI F, VENNERI MA, BIZIATO D, NONIS A, MOI D, SICA A, DI SERIO C, NALDINI L, DE PALMA M. (2009). A distinguishing gene signature shared by tumor-infiltrating Tie2-expressing monocytes, blood "resident" monocytes, and embryonic macrophages suggests common functions and developmental relationships. Blood 114: 901-914.

QIAN BZ, POLLARD JW. (2010) Macrophage diversity enhances tumor progression and metastasis. Cell 141: 39-51.

RAE F, WOODS K, SASMONO T, CAMPANALE N, TAYLOR D, OVCHINNIKOV DA GRIMMOND SM, HUME DA, RICARDO SD, LITTLE MH. (2007). Characterisation and trophic functions of murine embryonic macrophages based upon the use of a Csf1r-EGFP transgene reporter. Dev Biol 308: 232-246.

RAOS, LOBOVIB, VALLANCE JE, TSUJIKAWAK, SHIOJIMAI, AKUNURUS, WALSH K, BENJAMIN LE, LANG RA. (2007). Obligatory participation of macrophages in an angiopoietin 2-mediated cell death switch. Development 134: 4449-4458.

RIBATTI D. (2010) The seminal work of Werner Risau in the study of the development of the vascular system. Int J Dev Biol. 54: 567-572.

RODERO MP, KHOSROTEHRANI K. (2010) Skin wound healing modulation by macrophages. Int J Clin Exp Pathol. 3: 643-653.

RYMO SF, GERHARDT H, WOLFHAGEN SAND F, LANG R, UV A, BETSHOLTZ
C. (2011) A two-way communication between microglial cells and angiogenic sprouts regulates angiogenesis in aortic ring cultures. PLoS One 6:e15846.

SINDRILARU A, PETERS T, WIESCHALKA S, BAICAN C, BAICAN A, PETER H, HAINZLA, SCHATZS, QI Y, SCHLECHTA, WEISS JM, WLASCHEK M, SUNDERKÖTTER C, SCHARFFETTER-KOCHANEK K. (2011) An unrestrained proinflammatory $\mathrm{M} 1$ macrophage population induced by iron impairs wound healing in humans and mice. J Clin Invest. 121:985-997. DOI: 10.1172/JCI44490

SQUADRITO ML, DE PALMA M. (2011) Macrophage regulation of tumor angiogenesis: Implications for cancer therapy. Mol Aspects Med. 32: 123-145 (DOI:10.1016/j.mam.2011.04.005)

TAMMELA T, ALITALO K. (2010) Lymphangiogenesis: Molecular mechanisms and future promise. Cell 140: 460-476.

TONDRAVI MM, MCKERCHER SR, ANDERSON K, ERDMANN JM, QUIROZ M, MAKI R, TEITELBAUM SL. (1997). Osteopetrosis in mice lacking haematopoietic transcription factor PU.1. Nature 386: 81-84.

VAN KEMPEN LC, DE VISSER KE, COUSSENS LM. (2006). Inflammation, proteases and cancer. Eur J Cancer 42:728-734.

WELFORD AF, BIZIATO D, COFFELT SB, NUCERA S, FISHER M, PUCCI F, DI SERIO C, NALDINI L, DE PALMA M, TOZER GM, LEWIS CE. (2011) TIE2expressing macrophages limit the therapeutic efficacy of the vascular-disrupting agent combretastatin A4 phosphate in mice. J Clin Invest. 121:1969-1973.

WU Y, ZHAO RC, TREDGET EE. (2010) Concise review: bone marrow-derived stem/progenitor cells in cutaneous repair and regeneration. Stem Cells. 28:905-915

ZEISBERGER SM, ODERMATT B, MARTY C, ZEHNDER-FJALLMAN AH, BALLMER-HOFER K, SCHWENDENER RA. (2006). Clodronate-liposomemediated depletion of tumour-associated macrophages: a new and highly effective antiangiogenic therapy approach. Br J Cancer 95: 272-281.

ZIEGLER-HEITBROCK L, ANCUTA P, CROWE S, DALOD M, GRAU V, HART DN, LEENEN PJ, LIU YJ, MACPHERSON G, RANDOLPH GJ et al., (2010) Nomenclature of monocytes and dendritic cells in blood. Blood 116: e74-80. 


\section{Further Related Reading, published previously in the Int. J. Dev. Biol.}

The seminal work of Werner Risau in the study of the development of the vascular system Domenico Ribatti

Int. J. Dev. Biol. (2010) 54: 567-572

Over-expression of thymosin beta4 promotes abnormal tooth development and stimulation of hair growth Hee-Jae Cha, Deborah Philp, Soo-Hyun Lee, Hye-Sung Moon, Hynda K. Kleinman and Takashi Nakamura Int. J. Dev. Biol. (2010) 54: 135-140

Immune cells in the placental bed

Judith N. Bulmer, Paula J. Williams and Gendie E. Lash

Int. J. Dev. Biol. (2010) 54: 281-294

Critical growth factors and signalling pathways controlling human trophoblast invasion

Martin Knöfler

Int. J. Dev. Biol. (2010) 54: 269-280

The "chemoinvasion assay": a tool to study tumor and endothelial cell invasion of basement membranes Adriana Albini, Roberto Benelli, Douglas M. Noonan and Claudio Brigati

Int. J. Dev. Biol. (2004) 48: 563-571

The stroma reaction myofibroblast: a key player in the control of tumor cell behavior Alexis Desmoulière, Christelle Guyot and Giulio Gabbiani

Int. J. Dev. Biol. (2004) 48: 509-517

The chemokine network in cancer - much more than directing cell movement

Hagen Kulbe, Neil R. Levinson, Fran Balkwill and Julia L. Wilson

Int. J. Dev. Biol. (2004) 48: 489-496

Developmental biology of zebrafish myeloid cells.

Meredith O Crowhurst, Judith E Layton and Graham J Lieschke

Int. J. Dev. Biol. (2002) 46: 483-492

Tumor necrosis factor-alpha inhibits epithelial differentiation and morphogenesis in the mouse metanephric kidney in vitro.

C M Cale, N J Klein, G Morgan and A S Woolf

Int. J. Dev. Biol. (1998) 42: 663-674

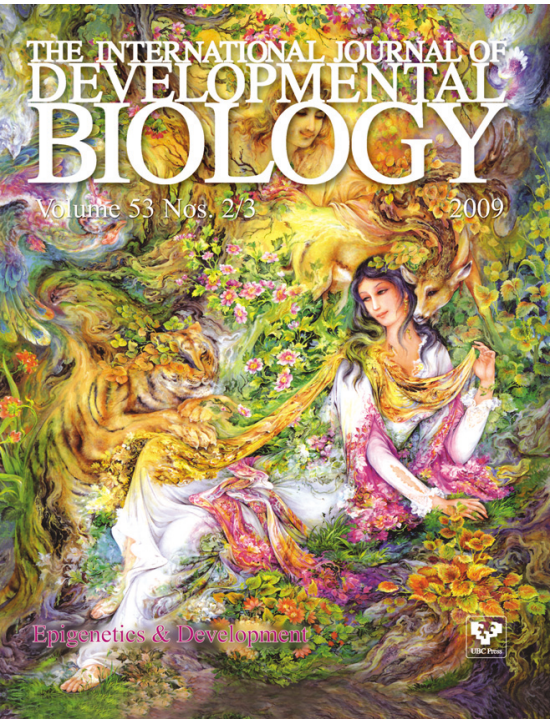

5 yr ISI Impact Factor $(2009)=3.253$

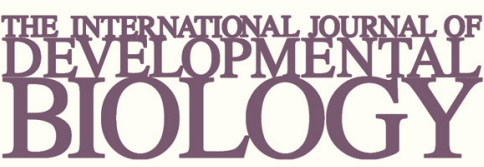

Volume 54 Nos. 6/7
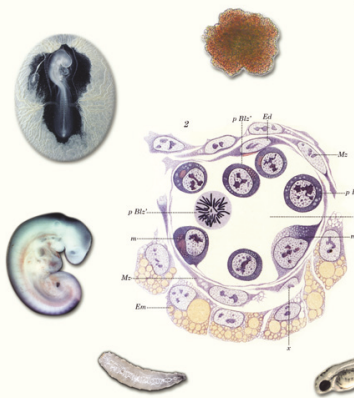

Developmental Hematopoiesis
Special Issue
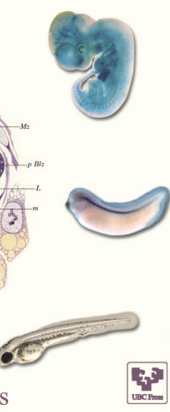

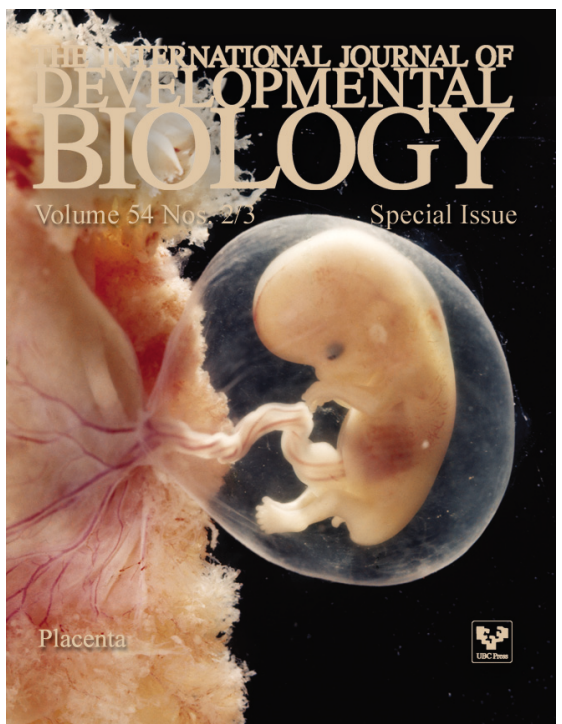

